\title{
Model-Based Design and Optimization of a Dielectric Elastomer Power Take-Off for Oscillating Wave Surge Energy Converters
}

\author{
Giacomo Moretti ${ }^{*}$, Marco Fontana*, Rocco Vertechy ${ }^{* \#}$ \\ *PERCRO-SEES, TeCIP Institute, Scuola Superiore di Studi Universitari e di Perfezionamento Sant'Anna, Pisa, \\ Piazza Martiri della Libertà, 56100, Pisa. \\ \# Department of Industrial Engineering, University of Bologna, Viale Risorgimento 2, 40136 Bologna, Italy
}

Corresponding Author:

Marco Fontana

e-mail: m.fontana@sssup.it

tel: +39050882516

fax: +39050882564

The total number of words of the manuscript, including entire text from title page to figure legends: 8500

The number of words of the abstract: 220

The number of figures: 13

The number of tables: 1 


\section{Abstract}

This paper investigates a new kind of device for producing electricity from the mechanical energy carried by ocean waves. The proposed machine, named Poly-Surge, is based on an existing sea-bottom hinged surging-flap concept that is equipped with a new Power Take-Off (PTO) system based on a novel soft Dielectric Elastomers (DEs) transducer.

DEs are highly deformable polymeric materials that can be used to conceive electrostatic generators relying on capacitance variation. This kind of generators shows a number of features that well match the requirements of a wave energy converter since they are light-weight, low-cost, tolerant to salty/aggressive marine environment, noise-free during operation, and easy to manufacture and install. The considered Poly-Surge converter employs a Parallelogram-Shaped DE Generator (PS-DEG) arranged in a dual agonist-antagonist configuration, which makes it possible to provide the flap with controllable bidirectional torques.

In this paper, first a complete wave-to-wire multiphysics model of the overall system is described that assumes a simplified hydrodynamic response for the hinged-flap and an electro-hyperelastic behaviour of the PS-DEG. Second, a procedure is presented for the dimensioning and optimization of the PS-DEG for given sets of PolySurge flap dimensions, wave-climate information and constraints on both design and operational variables. Finally, simulation results are provided to demonstrate that the Poly-Surge can achieve quasi-optimal power production with a properly designed agonist-antagonist DEG PTO system.

Keywords: Wave Energy, WEC, Oscillating Wave Surge Converter, Dielectric Elastomer, ParallelogramShaped Dielectric Elastomer Generator, Smart Materials, DEG, Poly-Surge, Polymeric PTO

\section{Introduction}

Ocean waves represent an abundant and widely available source of mechanical energy, and their exploitation for electric energy generation purposes has attracted the attention of the scientific community since the early Seventies [1]. In the last decade, a number of research works have investigated both theoretical and experimental aspects that are related to the assessment of wave energy resource and technology, and a number of reviews of the state of art have been published [2]-[4].

As of today, the commercial development of Wave Energy Converters (WECs) appears to be hindered by a number of techno-economic issues [2]. One of the most critical components for a WEC is the Power-Take-Off (PTO) unit, i.e. the subsystem that is responsible for the conversion between mechanical and electrical energy. Generally, state-of-art PTO systems are costly, poorly adaptable/controllable, subject to failure and presents scarce efficiency (especially in the low frequencies regimes that are typical of sea waves).

The employment of Dielectric Elastomers (DEs) in substitution of currently available PTO systems may represent an important step towards the implementation of cost effective WECs. DEs are highly deformable electrically insulating polymers that can be employed to conceive solid-state electro-mechanical transducers [5]. DEs are usually shaped in membranes (or films) that are pre-stretched and coated with compliant electrodes to form deformable electric capacitors, which can be employed as actuators, sensors and generators. In the latter mode of operation (namely, in a DE Generator (DEG)), the mechanical work done by an external entity is used to produce a reduction of transducer capacitance and, thus, to increase the electric potentials of charges that 
reside on its electrodes. In this manner, DEGs can convert the provided mechanical energy into electrostatic energy,

The employment of DEGs in energy harvesting applications is very promising [6], [7]; especially for ocean wave power extraction, where such transducers offer the following advantageous features: low cost, low weight, reduced number of moving parts, low noise, good resistance to impact loads and corrosion, and good energy conversion efficiency at typical operating frequencies of WECs.

In the last few years, a number of scientific works has been devoted to the study of DEGs for wave energy harvesting [8]. The Dutch company Single Buoy Mooring, for example, designed and built a prototype of flexible WEC, based on an elastomeric tube covered with ring-shaped DEGs and filled with pressurized sea water. The action of sea waves provoked the radial deformation of the tube and consequent electrical capacitance variation of the DEG rings [9]. Rosati Papini et al. [11] studied a WEC based on an Oscillating Water Column with a diaphragm-shaped DEG instead of a traditional air turbine PTO.

This paper introduces the Poly-Surge, a new Oscillating Wave Surge Converter (OWSC) equipped with a DEG PTO. OWSCs are near-shore WECs that employ a bottom-hinged flap (or paddle) featuring one rotational degree of freedom (referred to as pitch motion) around a pivot axis placed in proximity to the sea bed. While existing OWSC concepts are usually provided with hydraulic PTOs [14], the considered Poly-Surge relies on the use of one or more Parallelogram-Shaped DEGs (PS-DEGs) [15].

A sketch of the Poly-Surge is provided in Figure 1. As shown, PS-DEGs are rotary generators made by a parallelogram mechanism with an embedded DE membrane coated on both sides by compliant electrodes. In Poly-Surge, two neighbour links of the PS-DEG are respectively connected to the sea bed and to the paddle.

In a previous work [13], a Poly-Surge with one single PS-DEG (as depicted in Figure 1.a) has been investigated. In this paper, the improved DEG architecture (hereafter called Dual-DEG) depicted in Figure 1.b is considered which features two concurrent PS-DEGs that are located on opposite sides of the paddle. In the study, the possibility of offsetting the angular position of the PS-DEGs with respect to that of the flap is also considered.

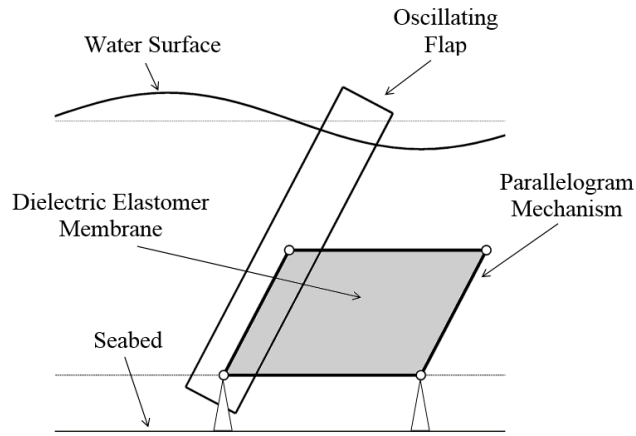

a)

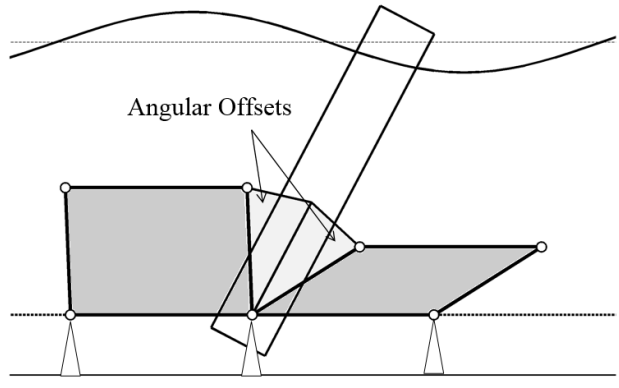

b)

Figure 1 - Schematic of Poly-Surge with a) single PS-DEG b) dual-DEG with offset.

In the following, Section II provides a simplified hydrodynamics model for the oscillating flap that relies on potential flow theory and Boundary Elements Method (BEM) codes. After presentation, this hydrodynamics model is used to investigate the optimal response that should be provided by a generic PTO in order to maximize the OWSC power capture in the presence of amplitude constraints of paddle motion. Section III describes an 
analytic model for the PS-DEG response and proposes a design procedure for a Dual-DEG PTO system that makes it possible to extract the desired optimal power output from the OWSC. Finally, a simulation case study is conducted in Section IV which considers an oscillating flap with selected dimensions, a hypothetic installation site with known wave climate and a specific DE material with given electromechanical properties and failure limits.

\section{Oscillating Wave Surge Converters}

OWSCs are near-shore devices, designed to operate in locations with 10-15 m of water depth [14]. Although shallow water waves are less energetic than offshore ones, the near-shore wave resource presents a narrower directional distribution. As a consequence, WECs operating in shallow water benefit of more constant available wave power in their orientation direction, which is only $10-20 \%$ less than the power available in $50 \mathrm{~m}$ deep water [16].

Two categories of OWSCs exist: with hinge located next to the sea bottom or with hinge located above the still water level. Considering that water particles velocity is very low next to the sea bed and is maximum at the free surface, hinging the flap at the bottom represents a better solution that maximizes the wave induced torque on the paddle and guarantees better energy conversion capability [17].

To date, some pre-commercial OWSC prototypes have been designed and manufactured and the most wellknown existing OWSC is the Oyster device, designed by Queen's University Belfast and by Aquamarine Power Ltd [14]. The prototype Oyster 1 consisted in a flap, made of five buoyant steel tubes, designed to completely cover the water column (from free-surface to sea-bed) in a reference water depth of $12 \mathrm{~m}$. The device is dimensioned to have a natural oscillating period of 20 s (i.e., larger than the period of typical sea waves) and is equipped with a hydraulic circuit that pumps pressurized water to an onshore power plant provided with hydraulic Pelton turbines. The design parameters have been chosen to maximize the wave-induced torque on the paddle and to limit maximum oscillation amplitude and speed (rather than to achieve resonance). This provides a WEC with a larger power capture, which is rather uniform in the range of wave frequencies the flap is expected to work within [14].

In the next sub-sections we introduce a dynamic model of the OWSC and study the optimal control for an ideal PTO under constrained oscillations of the paddle with a given limiting amplitude.

\subsection{Hydrodynamics model of the OWSC}

In this section, a simplified hydrodynamic model of the oscillating flap is presented. This model is based on a methodology which is commonly employed in the scientific literature to perform preliminary analyses and design of WECs [18]. Specifically, the equation that governs the dynamics of the primary mechanical interface of OWSCs (namely, the pitching flap) is:

$\left(I_{0}+I_{\infty}\right) \ddot{\theta}+\int_{0}^{t} \dot{\theta}(\tau) K(t-\tau) d \tau+k_{b} \theta=T_{w}(t)+T_{P T O}(t)$

where:

- $\theta$ is the angular position of the flap measured with respect to the vertical direction. 
- $I_{0}$ and $I_{\infty}$ are, respectively, the moment of inertia of the flap and the added moment of inertia at infinite oscillation frequency.

- $k_{b}$ is the hydrostatic stiffness of the flap and represents the combined/opposite effects of weight induced torque and buoyancy torque. In general, this resulting torque is not a linear function of $\theta$; here, $k_{b}$ derives from a linearization around the vertical equilibrium position $(\theta=0)$.

- the convolution integral accounts for the effect of the radiated waves generated by flap oscillations. In particular, $K$ is the convolution kernel that expresses the torque generated by radiated waves in response to an impulsive motion of the paddle. $K$ is expressed in Fourier frequency domain as

$\hat{K}(\omega)=\hat{B}_{r}(\omega)+i \omega\left(\hat{I}_{\text {add }}(\omega)-I_{\infty}\right)$

where $\hat{B}_{r}(\omega)$ and $\hat{I}_{a d d}(\omega)$ are the radiation damping and the added moment of inertia of the flap respectively.

- $T_{w}(t)$ and $T_{P T O}(t)$ are the wave excitation torque and the PTO torque respectively.

Equation (1) is a simplified time-domain model based on potential flow theory and on a linearization of the hydrodynamics of the flap, and it is strictly valid only for small values of the oscillation angle, $\theta$. More accurate results may be obtained using non-linear potential flow solvers or CFD codes; nonetheless, their employment would be excessively complex and time-consuming, so they are not recommended to perform iterative calculations, like those involved in the preliminary design, optimization and assessment of a WEC.

Although the hydrodynamics in equation (1) is linear, the time-domain model makes it possible to include in the study several non-linear effects. In particular, in order to optimize the WEC power capture in presence of oscillation amplitude constraints, a non-linear profile for $T_{P T O}$ is considered here.

For simplicity, monochromatic waves have been assumed; thus, $T_{w}$ reads as

$$
T_{w}(t)=\Gamma \sin (\omega t)
$$

where $\Gamma$ (a scalar quantity) is the wave induced torque amplitude. The time phase of $T_{w}$ has been set to zero without loss of generality.

The parameters $I_{\infty}, k_{b}$ and $\Gamma$ are typically calculated for any given system geometry (flap dimensions, water depth, wave length etc.) through a frequency-domain analysis using BEM software such as WAMIT.

The convolution integral in equation (1) is computationally inconvenient. A very common approximation for its solution is to resort to the following state-space differential system [20]:

$$
\left\{\begin{array}{c}
\int_{0}^{t} \dot{\theta}(\tau) K(t-\tau) d \tau \approx \mathbf{C} \boldsymbol{x} \\
\dot{\boldsymbol{x}}=\mathbf{A} \boldsymbol{x}+\mathbf{B} \dot{\theta}
\end{array}\right.
$$

where $\boldsymbol{x}$ is an internal state variable having proper dimension. In this work, $\boldsymbol{x}$ is chosen to be a three-dimensional vector. The matrices $\mathbf{A}, \mathbf{B}$ and $\mathbf{C}$ are calculated with a system identification procedure, like that described in [20], using the functions $\hat{B}_{r}(\omega)$ and $\hat{I}_{\text {add }}(\omega)$ that are obtained from the BEM analysis. 


\subsection{OWSC optimum control}

In this subsection, the optimal control of the OWSC is investigated. The control aims at maximizing the WEC power output in correspondence of each considered Sea State (SS).

For a generic SS with wave period $\tau$ (i.e., the angular frequency is $\omega=2 \pi \tau^{-1}$ ) and height $H$, the WEC power output is given by

$$
P=-\frac{1}{\tau} \int_{0}^{\tau} T_{P T O}(t) \dot{\theta}(t) d t
$$

If no constraints are present on both flap oscillation amplitude and maximum allowed torque, the optimal PTO torque is expressed by the so-called complex-conjugate control law [21] that is described in the following.

Using a frequency-domain formulation, equation (1) can be rewritten as

$$
\hat{Z}(\omega) \hat{\theta}(\omega)=\hat{T}_{w}(\omega)+\hat{T}_{P T O}(\omega)
$$

where $\hat{\theta}(\omega), \hat{T}_{w}(\omega)$ and $\hat{T}_{\text {PTO }}(\omega)$ are the Fourier transforms of $\theta(t), T_{w}(t)$ and $T_{P T O}(t)$ respectively, and $\hat{Z}(\omega)=k_{b}-\omega^{2}\left(I_{0}+\hat{I}_{\text {add }}(\omega)\right)+i \omega \hat{B}_{r}(\omega)$.

Then, the optimal PTO torque that maximises equation (5) can be found as $\hat{T}_{P T O, \text { opt }}(\omega)=\hat{Z}^{*}(\omega) \hat{\theta}(\omega)$, where $\hat{Z}^{*}(\omega)$ is the complex-conjugate of $\hat{Z}(\omega)$ [21]. This result implies that, if monochromatic waves are considered, the PTO torque can be expressed as a linear combination of factors that are proportional to $\theta$ and $\dot{\theta}$. Moreover, the corresponding optimal angular velocity of the flap results to be in phase with the excitation torque. In the frequency domain, this reads as

$$
\left.i \omega \hat{\theta}(\omega)\right|_{o p t}=\frac{\hat{T}_{w}(\omega)}{2 \hat{B}_{r}(\omega)} .
$$

Nevertheless, many practical cases require the introduction of some motion or torque constraint. In this article, the motion of the flap is assumed to be limited in amplitude, which reads as

$|\theta| \leq \theta_{\max }$

As pointed out in [18], the imposition of a constraint of this kind is reasonable due to the following issues.

- The hydrodynamic parameters, derived from BEM analysis, are usually calculated with reference to the flap being perpendicular to the seabed. Thus, the proposed model is rigorously valid only for small oscillations of the flap around the vertical position $(\theta=0)$.

- Large oscillations are characterized by large angular velocities, which induce vortex shedding and other non-linear phenomena that imply energy losses and are not accounted in the described dynamic model.

- In a real context, the motion of the flap is intrinsically limited. Indeed, as the pitch angle increases, the force on the paddle rapidly falls to zero (since it gets completely submerged, thereby perceiving less influence from surface waves).

In presence of constraint (9), the optimal profile of the function $T_{P T O}(t)$ that maximizes (5) can be numerically found as reported in [22], where a procedure is described for an oscillating buoy WEC with a semi-submerged spherical shape. Such a procedure can be easily generalized to any WEC whose dynamics can be described by equations (1) and (4). In [22], two strategies are proposed to optimize the WEC law of motion: by using the optimal velocity profile as unknown or by directly optimizing the function $T_{P т O}(t)$. In the present work, the first 
strategy is assumed, since it provides more accurate results with lower computational effort. The optimization problem is approached by selecting a sufficiently large time horizon and discretizing it with an appropriate timestep. In this way, the optimization can be reduced to a minimization problem of a constrained quadratic objective function that can be solved numerically with conventional algorithms. The output of such a minimization is an optimal velocity profile that allows the calculation of the optimal displacement (by integration), acceleration (by derivation) and the PTO torque profile (using equation (1)).

As alternatives, other approaches are available to determine the optimal dynamics of a constrained WEC; for instance, a solution can be obtained using the formalisms of calculus of variations [23].

As an example, Figure 2 shows numerical results relative to a selected flap geometry and sea wave conditions. A constrained oscillation amplitude $\theta_{\max }=\pi / 6$ was considered and a time-step of $0.04 \mathrm{~s}$ was used to discretize the time horizon. Figure 2.a reports the optimal profiles of angular position and velocity, while Figure 2.b shows the wave excitation torque and the optimal PTO torque.

As shown in Figure 2.a, the obtained solution is characterized by a latching-like behaviour, i.e. the device is kept fixed in correspondence of the maximum displacement position (namely, $\theta= \pm \theta_{\max }$ ) and released at specific time instants. At the extremities of such intervals, the flap velocity profile has vertical tangent (which corresponds to an infinite acceleration) and the resulting PTO torque presents, theoretically, infinite peaks, as also observed in [24] (see Figure 2.b).

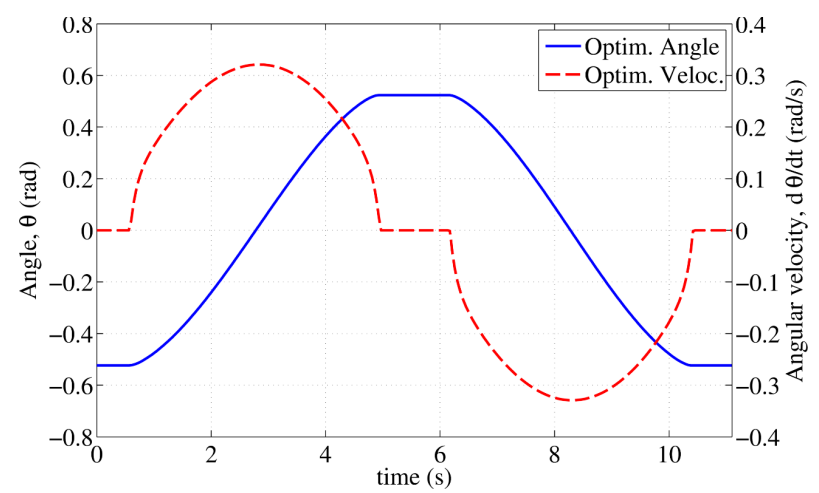

(a)

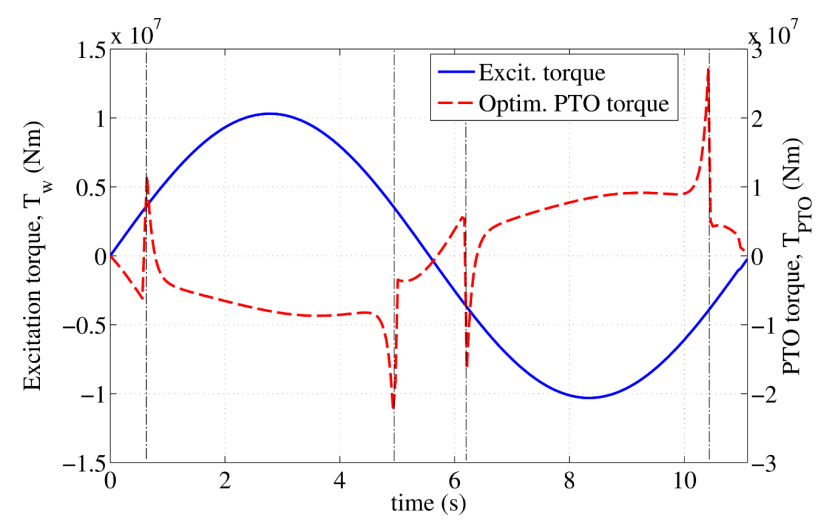

(b)

Figure 2 - a) Optimal flap velocity and angular position, b) Excitation torque and optimal PTO torque profiles over a wave period for a reference pitching flap. Paddle dimensions are: $11 \mathrm{~m}$ height, $18 \mathrm{~m}$ width and $2 \mathrm{~m}$ thickness. Oscillation amplitude is limited to $\pi / 6$. An incident wave with $H=3.28 \mathrm{~m}$ and $\tau=11.1 \mathrm{~s}$ is considered. Peaks in $T_{P T O}$ asymptotically tend to infinite as the chosen discretization time-step tends to zero. 
A comparison of Figure 2.a and Figure 2.b shows that the optimum flap angular velocity is in phase with the excitation torque, just as in the solution of the unconstrained problem (see equation (8)).

Since $\theta(t)$ and $T_{\text {РTO }}(t)$ are periodic functions of time, the PTO torque-displacement responses (in presence of different SSs) can be represented by closed lines on the $\theta-T_{P T O}$ plane. This type of representation is useful for the design of specific PTOs, like those based on DEGs, that have a torque response intrinsically expressed as a function of position [25]. The $\theta-T_{P T O}$ control trajectory for the discussed example is reported in Figure 3 , in which we can observe the theoretically infinite peak torques occurring in correspondence of the extreme positions $\theta= \pm \theta_{\max }$

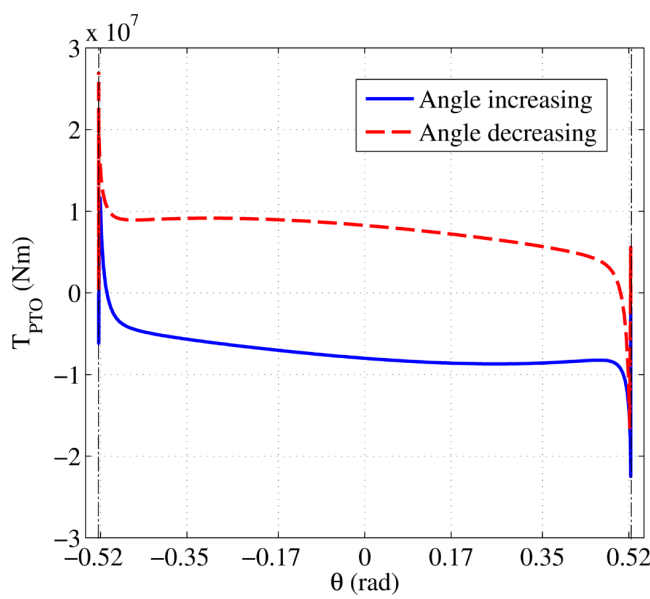

Figure 3 - Profile of optimal PTO torque as a function of flap angular position, $\theta$, obtained by combining the data of Figure 2.a and Figure 2.b.

\section{Model of the Parallelogram-Shaped Dielectric Elastomer Generator}

In this section, a mathematical model for the PS-DEG is provided with the aim of predicting the torque response $\left(T_{P T O}\right)$ of the DEG as a function of its position and electric activation. Such a model, which has been proposed and validated in a previous work [15], is employed here to study how the PS-DEG response can be coupled to the OWSC dynamics.

The PS-DEG is a rotary generator which exploits cyclic deformation and electric activation of one or more DE layers with double-side coating of compliant electrodes. A possible multi-layered structure for a PS-DEG is shown in Figure 4.a. Using this solution instead of a single DE layer allows to reduce the activation voltage of the membranes and guarantees better homogeneity of the electric field within the layers.

Dielectric materials that can be effectively used to build the PS-DEG are Natural Rubber (NR), silicone and acrylic elastomers. Deformable electrodes can be made by carbon powder or grease, liquid films, metallic thin films or conductive elastomeric layers [5].

From a functional standpoint, the PS-DEG is a variable capacitor which can be used as a charge pump to convert the mechanical work, performed on the DEG by external forces, into electrostatic energy, that is stored in the electric field within the capacitor or is removed from its terminals by means of an external electrical circuit.

The PS-DEG is an interesting generator concept because: 1) it can be easily coupled with systems featuring one degree of freedom and reciprocating rotary motion; 2) the presence of the parallelogram mechanism around the edges of the membrane guarantees homogeneous deformation. 
With reference to the Poly-Surge architecture depicted in Figure 1.b, the geometric configuration of the generator is uniquely identified by means of the variable $\theta$ (assumed positive in the clockwise direction), which represents the angular displacement of the flap with respect to the vertical direction. Since the system is antisymmetric, we can simplify the analysis by considering only one of the two PS-DEGs. Referring to Figure 4.b, the generator has one link, with length $l_{l}$, parallel to the horizontal plane and one link, with length $l_{2}$, rigidly connected to the flap by means of a rigid plate and forming an angle $\theta+\theta_{\text {off }}$ with the vertical direction $\left(\theta_{\text {off }}\right.$ being an offset angle). The DE membrane is mounted on the frame with a certain degree of biaxial pre-stretch, which is mathematically identified by the pre-stretches along principal directions $\xi$ and $\eta$ of deformation (namely, along the bisectors of the parallelogram angles, see Figure 4.b). Pre-stretches need to be referred to a specific configuration. In this article, they are referred to the configuration identified by $\theta=-\theta_{\text {off }}$, in which the angles of the parallelogram are $90^{\circ}$. Such a configuration is not necessarily included in the effective operation range of the DEG, but it is always possible to mathematically refer the pre-stretches to this position. In particular, we call $\lambda_{l p}$ and $\lambda_{2 p}$ the pre-stretches along $\xi$ and $\eta$ respectively.

As the parallelogram changes configuration, the stretch field within the DE membrane varies but remains spatially uniform (see Figure 4.b).

With reference to Figure 4.b, indicating with $\overline{\mathrm{AF}}$ and $\overline{\mathrm{DE}}$ the lengths of the bisectors of angles DÂB and $\mathrm{ADC}$ respectively (with $\overline{\mathrm{AF}}_{0}$ and $\overline{\mathrm{DE}}_{0}$ indicating their values in the reference configuration for which $\theta=-\theta_{\text {off }}$ ), the first and second principal stretches of the mounted membrane (namely, $\lambda_{1}$ and $\lambda_{2}$ ) can be expressed as function of $\theta$ via the following relations:

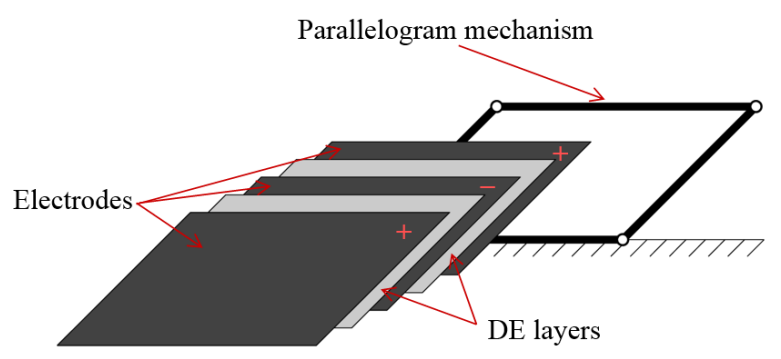

(a)

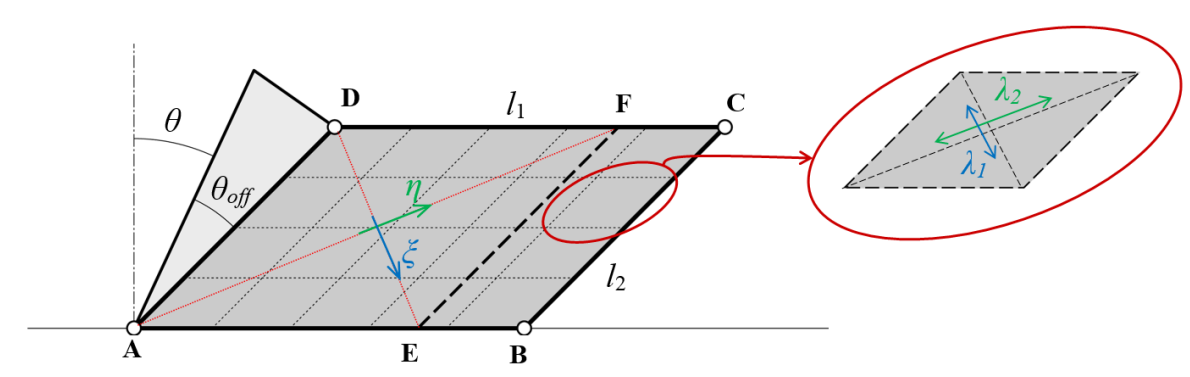

(b)

Figure 4 -a) Exploded view drawing of the PS-DEG with stacked dielectric layers and electrodes; b) Parallelogram-Shaped Dielectric Elastomer Generator (PS-DEG) with offset plate. With reference to angles DÂB and ADFF, the local reference frame $\xi-\eta$ lies along the bisectors ( $\overline{\mathrm{AF}}$ and $\overline{\mathrm{DE}}$ respectively) and is a principal deformation system. Deformation is supposed spatially uniform. 
$\lambda_{1}=\lambda_{1 p} \frac{\overline{D E}}{\overline{D E}_{0}}=\sqrt{2} \lambda_{1 p} \sin \left(\frac{1}{2}\left(\frac{\pi}{2}-\theta-\theta_{\text {off }}\right)\right), \lambda_{2}=\lambda_{2 p} \frac{\overline{A F}}{\overline{A F}_{0}}=\sqrt{2} \lambda_{2 p} \cos \left(\frac{1}{2}\left(\frac{\pi}{2}-\theta-\theta_{\text {off }}\right)\right)$

where $\lambda_{1 p}$ and $\lambda_{2 p}$ indicate the prestretches of the DE membrane prior to its mounting on the frame. Since the dielectric elastomer material is assumed incompressible [26], the stretch in thickness direction is

$\lambda_{3}=\left(\lambda_{1} \lambda_{2}\right)^{-1}$.

The torque provided by the PS-DEG can be calculated by means of an energy balance. It is assumed that when the generator is electrically activated, charge on the electrodes and electric field within the DE layers are spatially uniform.

The physical state of the PS-DEG in any configuration is completely represented using two variables. In this section, the pitch angle, $\theta$, and the electric field, $E$, are used as state variables. The charge, $Q$, on the capacitor electrodes or the voltage, $V$, applied between the electric terminals of the DEG may be used in place of $E$.

Considering that the PS-DEG capacitance is given by

$C=\varepsilon \Omega^{-1}\left(l_{1} l_{2} \cos \left(\theta+\theta_{\text {off }}\right)\right)^{2}$,

where $\varepsilon$ is the dielectric permittivity of the considered DE material and $\Omega$ is the total membrane volume, the PSDEG voltage $V$ and charge $Q$ can be expressed as functions of $\theta$ and $E$, namely

$V=E \Omega\left(l_{1} l_{2} \cos \left(\theta+\theta_{\text {off }}\right)\right)^{-1}, Q=C V=\varepsilon E l_{1} l_{2} \cos \left(\theta+\theta_{\text {off }}\right)$.

In this paper, the following effects are neglected: 1) the inertial and gravitational forces acting on the polymeric membrane and on the mechanism links; 2) friction at the joints of the mechanism; 3) visco-elastic losses due to mechanical hysteresis of the considered DE material; 4) electric losses, such as leakage currents through the DE layers and charge dispersion from the electrodes; 5) losses of electrical equipment (cables, electronic components, balance of plants, etc.).

With these assumptions, for an infinitesimal variation $d \theta$ of the pitch angle $\theta$, the energy balance of the PS-DEG reads as

$d U_{e}+d U_{m}=d W_{e}+d W_{m}$,

where $d W_{m}$ and $d W_{e}$ are the infinitesimal works performed on the membrane by the external torque (applied between two adjacent links of the parallelogram mechanism) and by the external electric circuit respectively, while $d U_{e}$ and $d U_{m}$ are the infinitesimal variations of the electrostatic potential energy and of the elastic potential energy stored in the DE membrane.

The potential energy terms read as

$U_{e}=\varepsilon \Omega E^{2} / 2, U_{m}=\Omega \Psi(\theta)$,

where $\Psi$ is the strain-energy function (or Helmholtz free-energy function), that expresses the amount of elastic potential energy stored in a unitary volume of deformed DE material [26]. Several constitutive models exist that relate $\Psi$ to the principal stretches of the membrane [27]. In this paper, we consider a hyperelastic model in Gent form [13], namely

$\Psi=-a \ln \left(\left(I_{m}-\lambda_{1}^{2}-\lambda_{2}^{2}-\lambda_{1}^{-2} \lambda_{2}^{-2}\right) /\left(I_{m}-3\right)\right)$,

where $a$ and $I_{m}$ are constitutive parameters of the considered DE material.

Indicating with $T_{D E G}$ the torque provided by the DEG to the flap, the mechanical and electrostatic works result in 
$d W_{m}=-T_{D E G} d \theta, d W_{e}=V d Q$.

Using equations (13), $d W_{e}$ can be expressed as a function of $\theta$ and $E$, namely

$d W_{e}=\varepsilon \Omega E d E-\varepsilon \Omega E^{2} \tan \left(\theta+\theta_{\text {off }}\right) d \theta$.

Combining equations (14) - (17), the following analytical expression for $T_{D E G}$ is obtained

$T_{D E G}=T_{D E G, m}+T_{D E G, e}$,

where $T_{D E G}$ has been split into the following elastic and electrostatic terms

$T_{D E G, m}=-\Omega \frac{d \Psi}{d \theta}, T_{D E G, e}=-\varepsilon \Omega E^{2} \tan \left(\theta+\theta_{o f f}\right)$.

The amount of electric energy generated (or spent) in correspondence of displacement $d \theta$ results in

$d U_{e}-d W_{e}=\varepsilon \Omega E^{2} \tan \left(\theta+\theta_{o f f}\right) d \theta$.

Notice that electric energy can be generated or stored (namely, expression (21) is positive) only if $\tan \left(\theta+\theta_{\text {off }}\right) d \theta>0$; that is, when the membrane surface (and, thus, its capacitance) decreases. On the contrary, the DE transducer can behave as an actuator, thereby absorbing electrical energy from the external circuit and converting it into mechanical work. It will be shown in this paper that, when a DEG is coupled to a WEC, it may be convenient to make it work as actuator (not only as generator) for a part of its operating cycle, as this may provide a more favourable dynamics of the flap, thus improving the total power capture.

Equations (20) and (21) highlight that the PS-DEG torque response and the amount of converted energy depend on the overall amount of DE material volume, $\Omega$, only, and do not depend on specific geometric parameters of the mechanism, like the parallelogram aspect ratio $l_{l} / l_{2}$. This makes it possible to choose such parameters according to other requirements imposed by the application of Poly-Surge. For example, the best layout may be chosen taking into account functional aspects (such as encumbrances, mass distribution, influence of PS-DEG on overall Poly-OWC hydrodynamics) and/or technological issues (such as manufacturing, assembly, installation).

\subsection{Failure mechanisms and operation constraints}

This sub-section analyses failure mechanisms of the PS-DEG in order to define feasible operating ranges of the generator. According to [7] and [15], the considered constraints are: 1) membrane rupture due to material overstretching; 2) electrical break-down due to an excessive applied electric field; 3) electro-mechanical buckling, that is, loss of tension of the DE membrane layers. Condition 1) represents a purely mechanical failure mechanism, condition 2) is a purely electrostatic failure mechanism, while buckling 3) is due to a combined electro-mechanical instability phenomenon.

\section{Mechanical rupture}

Limitations in the maximum deformations of the DE membrane are determined by mechanical rupture. In this paper, Kawabata's criterion [28] is assumed, i.e., mechanical rupture in the $i$-th principal direction occurs if

$\lambda_{i}>\lambda_{u}, \quad$ with $i=1,2,3$.

Here, $\lambda_{u}$ is the rupture stretch and is a constant that depends only on the considered DE material. 


\section{$\underline{\text { Electric break-down }}$}

Break-down of a dielectric medium occurs if the electric field exceeds a limit value, provoking a permanent damage of the material. Assuming a constant value for the break-down field, $E_{B D}$, and electric break-down occurs if

$E>E_{B D}$.

Expression (23) represents a simplified but conservative condition, since dielectric strength may vary (increase) with the strain field of the material [15].

\section{Electro-mechanical buckling}

In order to work properly, the DE membrane must remain in tension during its operation. If the total electromechanical stresses in the plane of the DE membrane assume negative values, the material wrinkles and the transduction mechanism fails. The total stress in a generic direction is the result of an elastic contribution, induced by the stretch, and an electrostatic contribution due to electric activation.

Referring to the principal stresses in the plane of the membrane [26], loss of tension occurs if

$\sigma_{1}=\sigma_{m 1}-\sigma_{e}=\lambda_{1} \frac{\partial \Psi}{\partial \lambda_{1}}-\sigma_{e}<0$ or $\sigma_{2}=\sigma_{m 2}-\sigma_{e}=\lambda_{2} \frac{\partial \Psi}{\partial \lambda_{2}}-\sigma_{e}<0$,

where $\sigma_{m 1}$ and $\sigma_{m 2}$ are the principal elastic stresses due to membrane deformation (with $\lambda_{1}, \lambda_{2}$ and $\Psi$ being given by equations (10) and (16)), whereas $\sigma_{e}$ is a purely electrostatic stress given by $\sigma_{e}=\varepsilon E^{2}$, as demonstrated in [6].

\subsection{The Dual-DEG and its operating space}

In this subsection, an agonist-antagonist layout for the PS-DEG is introduced and analysed. The mathematical model described so far is extended to the dual-DEG configuration and the operative space of the resulting DEG (in terms of angular position and supplied torque) is studied.

A dual-DEG consists in two identical generators concurrently mounted on the right and left side of the flap midline axis, as shown in Figure 5.

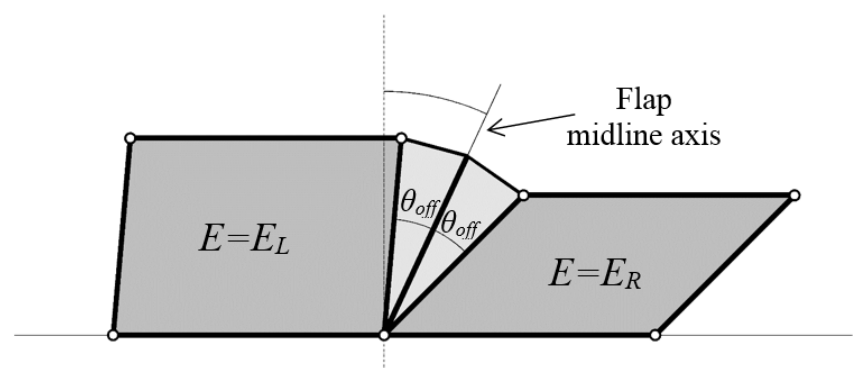

Figure 5 - Agonist-antagonist layout for a couple of Parallelogram-Shaped Dielectric Elastomer Generators

The torque $T_{D E G}(\theta, E)$ of a single PS-DEG is given by equations (19) and (20). In presence of an agonistantagonist configuration, the total torque of the generator reads as

$T_{D E G, t o t}=T_{D E G}\left(\theta, E_{R}\right)-T_{D E G}\left(-\theta, E_{L}\right)$, 
where both $\theta$ and $T_{D E G, t o t}$ are assumed positive in the clockwise direction, while $E_{R}$ and $E_{L}$ respectively represent the electric field across the PS-DEGs located on the right and on the left side of the flap.

In a generic position, $\theta$, the dual-DEG can provide torques that can be adjusted by properly selecting the values of the electric fields $E_{R}$ and $E_{L}$.

For a given choice of the design parameters, the operating space of the Dual-DEG can be represented in a torque-displacement diagram and is limited by the following curves:

- The torque-displacement curves relative to the separate activation of the two PS-DEGs with the maximum allowed electric field, i.e. $\left[E_{L}=E_{B D} \& E_{R}=0\right]$ or $\left[E_{L}=0 \& E_{R}=E_{B D}\right]$.

- The vertical straight lines identifying the angles at which mechanical rupture occurs.

- The curves relative to the loss of tension condition.

To provide an example, Figure 6 reports the useful operating space of a Dual-DEG featuring the following geometric and material characteristics:

- Polymer volume (total left and right PS-DEGs): $\Omega=24.6 \mathrm{~m}^{3}$.

- Offset angle: $\theta_{\text {off }}=40^{\circ}$.

- Pre-stretches: $\lambda_{1 p}=4.2, \lambda_{2 p}=3.9$.

- Mechanical and electrical properties given in Table I.

In the Figure, the operating domain of the dual-DEG is represented by the dotted surface.

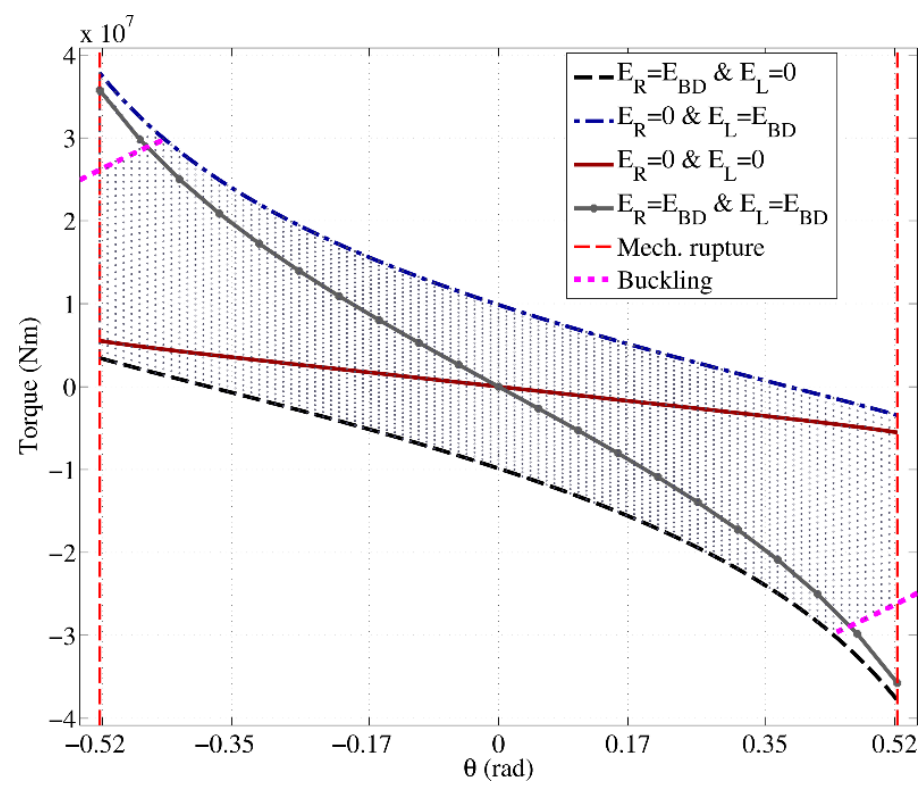

Figure 6 - Example of the operational space of the dual-DEG in the torque-position plane. The dotted surface represents the allowed mechanical states for the generator.

\section{Design and control of a polymeric PTO for OWSCs: numerical case study}

In this section, a numerical example is presented with the aim of providing preliminary design and control criteria for OWSCs having known geometry, equipped with a Dual-DEG PTO and working in a given wave climate. 
The proposed design approach aims at providing a PS-DEG PTO that is able to operate implementing the WEC controller presented in Section II, i.e., a PS-DEG whose torque-position characteristic is matched to those required by the OWSC to optimally extract energy in a given set of sea states conditions.

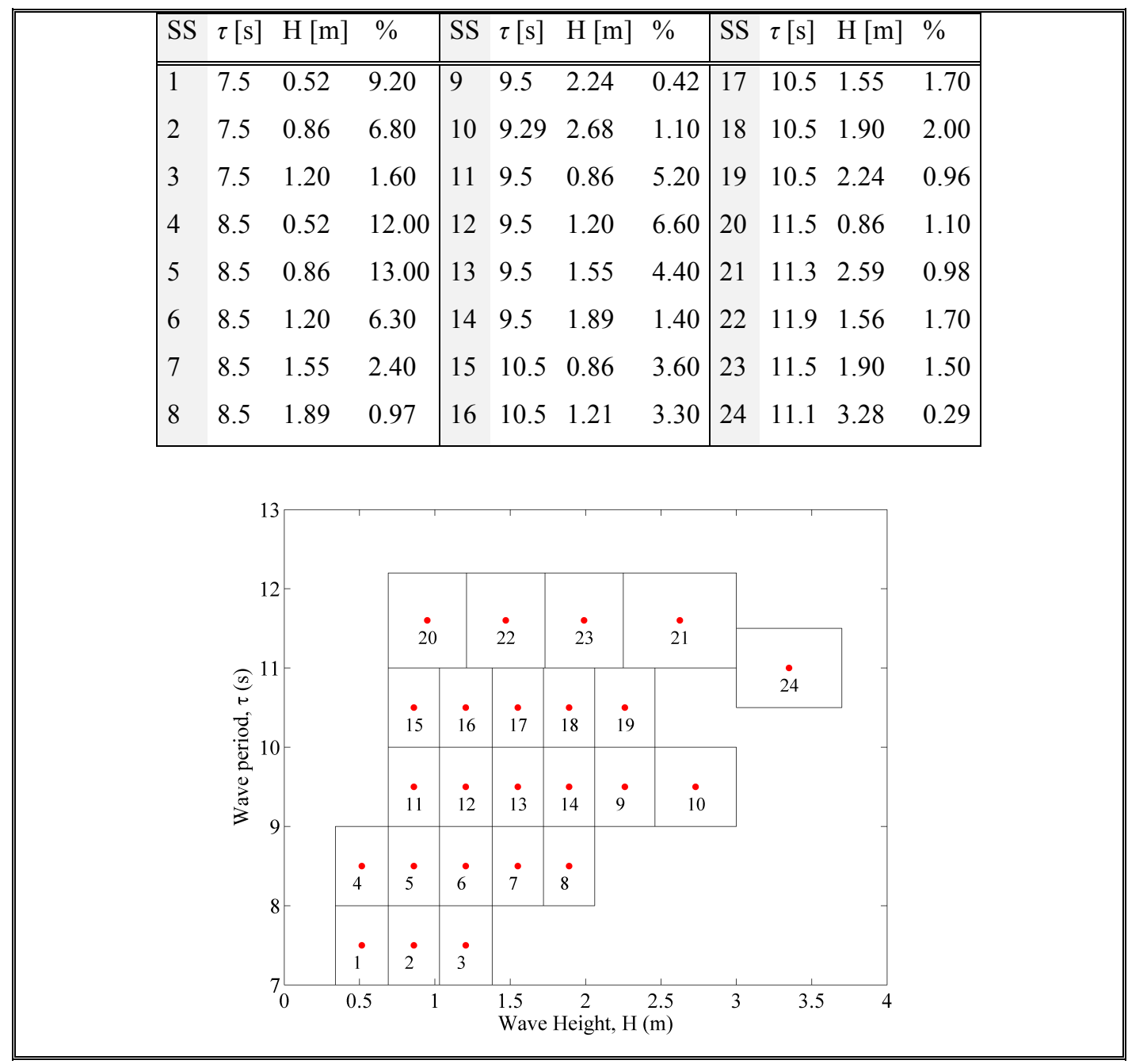

Figure 7 - Scatter matrix for the reference site. Regular wave data for each Sea State (SS) are expressed in terms of wave period, height and frequency of occurrence.

As for the flap geometry, the dimensions provided in [13] and [14] are considered in order to be able to make comparisons. The reference flap is a parallelepiped with a height of $12 \mathrm{~m}$, a width (assumed parallel to the wave front) of $18 \mathrm{~m}$ and a thickness of $2 \mathrm{~m}$. The flap is installed in a $12 \mathrm{~m}$ nominal depth, it has a draft of $10 \mathrm{~m}$ and a pivot submerged at a depth of $9 \mathrm{~m}$. Flap density is assumed to be homogeneous and equal to $300 \mathrm{~kg} \cdot \mathrm{m}^{-3}$. According to previous studies [18], the limitation on the maximum allowed pitch angle (see equation (8)) is set to $\theta_{\max }=\pi / 6$.

A near-shore intermediate-depth site off Pico Island (Azores) has been used as reference installation location. This site is of particular interest because it presents good available resource even in relatively shallow water thanks to its peculiar geographic location (open ocean, about $1300 \mathrm{~km}$ far from the Portuguese coast) [29]. The considered reference wave climate is described by 24 monochromatic regular SSs (resumed in Figure 7) with known occurrence frequency, as proposed in [13]. 


\subsection{Target PTO control trajectories for the reference OWSC}

For each of the SS, the optimal torque profile that the PTO is required to supply can be found by applying the methodology outlined in Section 2.2. However, the obtained results yield a PTO response featuring overshoots with an infinite value of the required torque.

Infinite impulsive loads are not physically realizable with real generators. In addition, the presence of large (even finite) load peaks should be in general avoided since: 1) they are responsible for irregular operation, with severe vibrations and possible mechanical failure of PTO parts; 2) the generator should be oversized (thus being more massive and costly) in order to provide and resist such loads.

In order to avoid this inconvenient, torque peaks have been mitigated by smoothing the optimal position profiles through a moving average filter. For each SS, the span of the filter is set to the maximum value that generates a reduction of the power output lower than $0.2 \%$ with respect to the optimal power output with no filters.

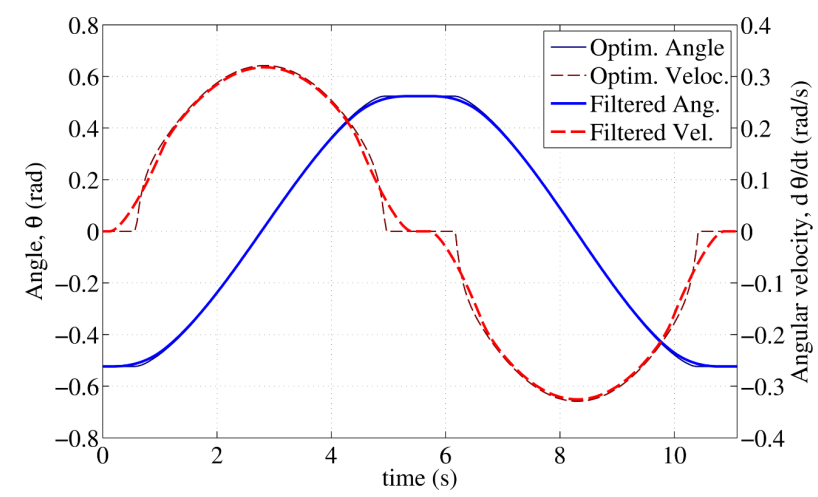

(a)

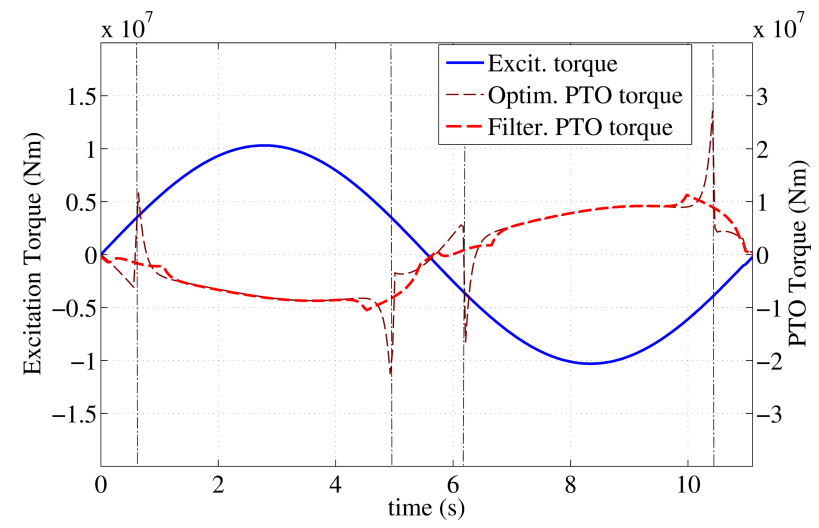

(b)

Figure 8 - Required PTO characteristic for the sea state SS24: a) Optimal and sub-optimal (filtered) profiles of angular position and velocity; b) wave excitation torque, optimal and smoothed PTO torque profiles. Notice that, in order to produce small variations in the position and velocity profiles, significant variations of the required PTO torque profiles are needed. Optimal datasets were calculated using a time-step of 0.04 s (i.e., 278 samples per dataset). Filtered profiles were obtained using a filter span of 23 samples (i.e., filtered values are calculated using a time window with $0.88 \mathrm{~s}$ width).

Figure 8.a depicts the optimal and filtered angular position time-series and the resulting angular velocity profiles required by the PTO for the sea state SS24. For the same SS, Figure 8.b shows the wave induced torque and the required PTO torque for both the optimal and filtered (non-optimal) cases. Notice that the power output reduction is very low while the torque peaks are consistently reduced (provided that the corresponding power reduction is less than $0.2 \%$ ); 


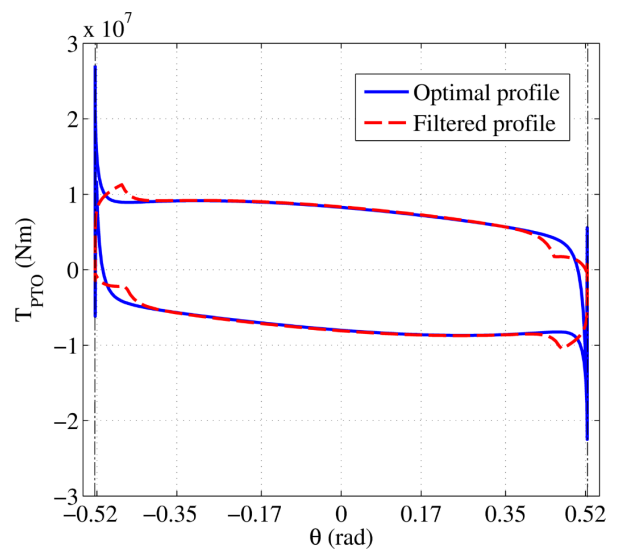

Figure 9 - Optimal and sub-optimal control trajectories on the $\theta-T_{\text {РТО }}$ plane for the SS24.

The torque-displacement characteristic required from the PTO for the SS24 is represented in Figure 9 for both the optimal and the filtered cases. The described procedure is applied to all of the sea states given in Figure 7. Notice that the optimization in [22] and latching-type optimum control are strictly required only for those wave conditions that would intrinsically induce flap oscillation amplitudes larger than $\theta_{\max }$. For the weakest sea states, the flap oscillation amplitude is intrinsically lower than $\theta_{\max }$ and the optimal PTO torque profiles are given by the complex-conjugate control [21] that was detailed in Section 2.2.

In Figure 10, we summarize the performances of the considered OWSC while operating under such a smoothed controller. In particular, for each SS:

- $\quad$ Figure 10.a shows the power matrix expressing the Poly-Surge power output in kW;

- Figure 10.b reports the capture factor, defined as the ratio between the WEC power output and the incident ocean power that is carried by a wave front having the same width of the device.

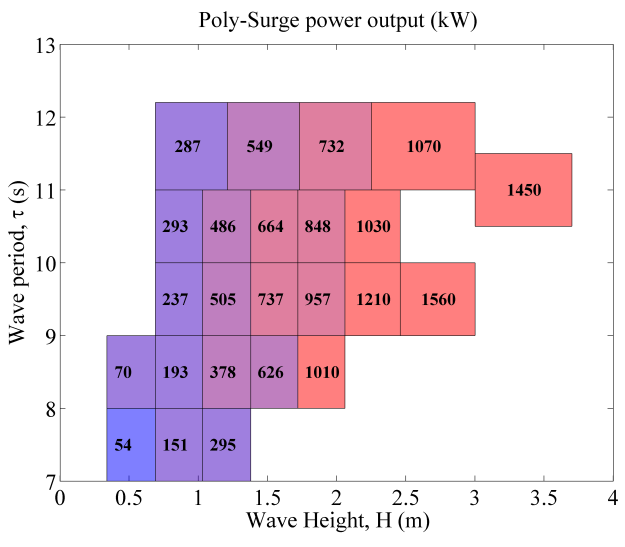

a)

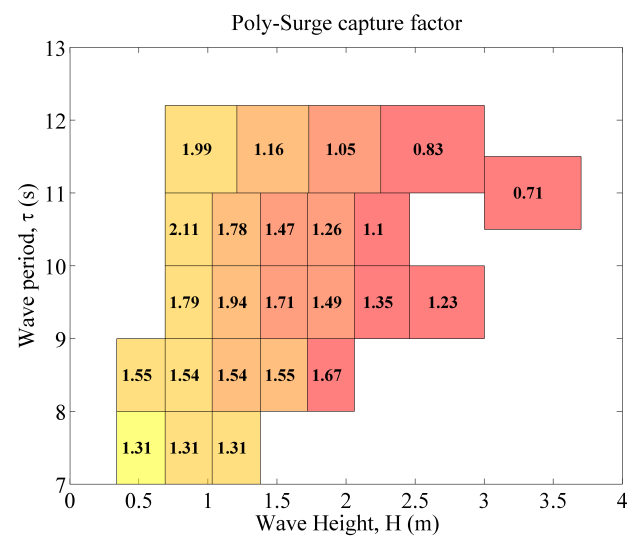

b)

Figure 10 - Power matrix a) and capture factor matrix b) for all of the considered sea states.

As shown in Figure 10.b, the capture factor is often larger than one. This is a common result in this kind of analyses [18] since the OWSC behaves as a point absorber, as its width is much shorter than the wavelength, so it can capture energy from a larger wave front than its width [14]. Nonetheless, it should be remarked that this analysis uses monochromatic waves, which generally leads to an overestimation of the power output [19] and, thus, of the capture factor. 
From the power outputs reported in Figure 10.a and given the relative occurrence frequencies of the sea states detailed in Figure 7, the electrical energy that can be produced in a year (namely, 8760 operating hours) by the considered OWSC results in $2.79 \mathrm{GWh}$.

In order to design a Dual-DEG that is able to harvest such a large amount of energy, it is useful to plot in a same graph the required $\theta-T_{\text {PTO }}$ control curves for all of the considered sea states (see Figure 11). In fact, the DualDEG to be designed should feature a $\theta-T_{P T O}$ characteristic that fully envelopes the depicted set of control curves. For the reference case study, such an envelope is fully identified by only two of the reference control trajectories (marked with thick dotted lines in Figure 11).

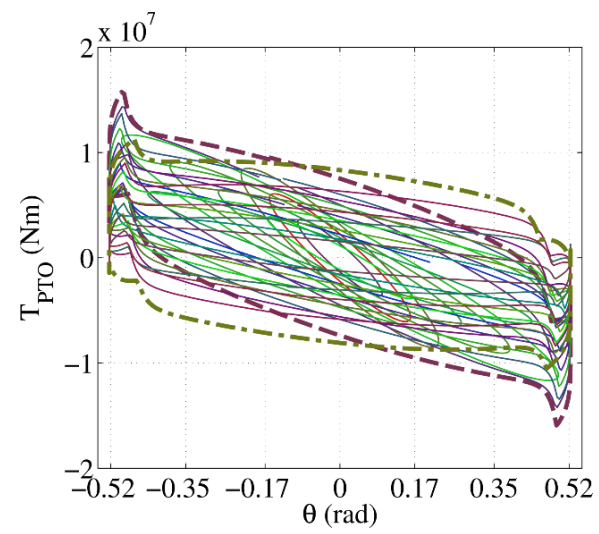

Figure 11 - Torque-displacement control curves for all the considered sea wave conditions. Thick dotted lines are relative to the sea states whose optimal control curves fully describe the operational space required by the PTO to be designed.

\subsection{Polymeric PTO design parameters optimization}

In this subsection, we investigate a design for a dual-DEG PTO having a torque-angle characteristic (see Figure 6) that satisfies the requirements detailed in the previous paragraphs and that fully envelop the control trajectories reported in Figure 11.

The study is conducted by considering a reference DE membrane made of NR whose electro-mechanical properties are listed in Table I (similar properties have also been used in [13]).

Table I- Electrical and mechanical properties of the reference NR elastomer.

\begin{tabular}{|l|l|}
\hline Dielectric strength (or break-down electric field) & $E_{B D}=200 \mathrm{MV} / \mathrm{m}$ \\
\hline Dielectric permittivity & $\varepsilon=2.7 \cdot 8.85 \mathrm{e}-12 \mathrm{~F} / \mathrm{m}$ \\
\hline Hyperelastic parameters (Gent model) & $a=2.5 \mathrm{e} 7 \mathrm{~Pa} ; I_{m}=116$ \\
\hline Uniaxial rupture stretch & $\lambda_{u}=5.5$ \\
\hline
\end{tabular}

The unknown design parameters of the Dual-DEG are:

- angular offset, $\theta_{\text {off; }}$;

- membrane pre-stretches, $\lambda_{l p}$ and $\lambda_{2 p}$;

- overall volume, $\Omega$, of DE material.

An iterative procedure has been used to identify a combination of these parameters that provides a DEG with the required $\theta-T_{\text {PTO }}$ characteristic while requiring the minimum amount of DE material (volume). 
In the optimization, the addition of an external torsion stiffness $k_{S}$ (that can be either positive or negative in value) has also been considered. This stiffness can be provided by means of a properly designed passive mechanical component, i.e. a torsion spring or an equivalent compliant mechanism. When included, the value of $k_{S}$ is also optimized. Figure 12 provides the results of the analysis, which includes:

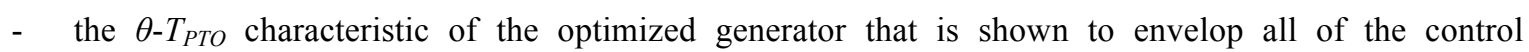
trajectories that are required to maximize overall energy productivity;

- $\quad$ the optimal design parameters of the Dual-DEG.

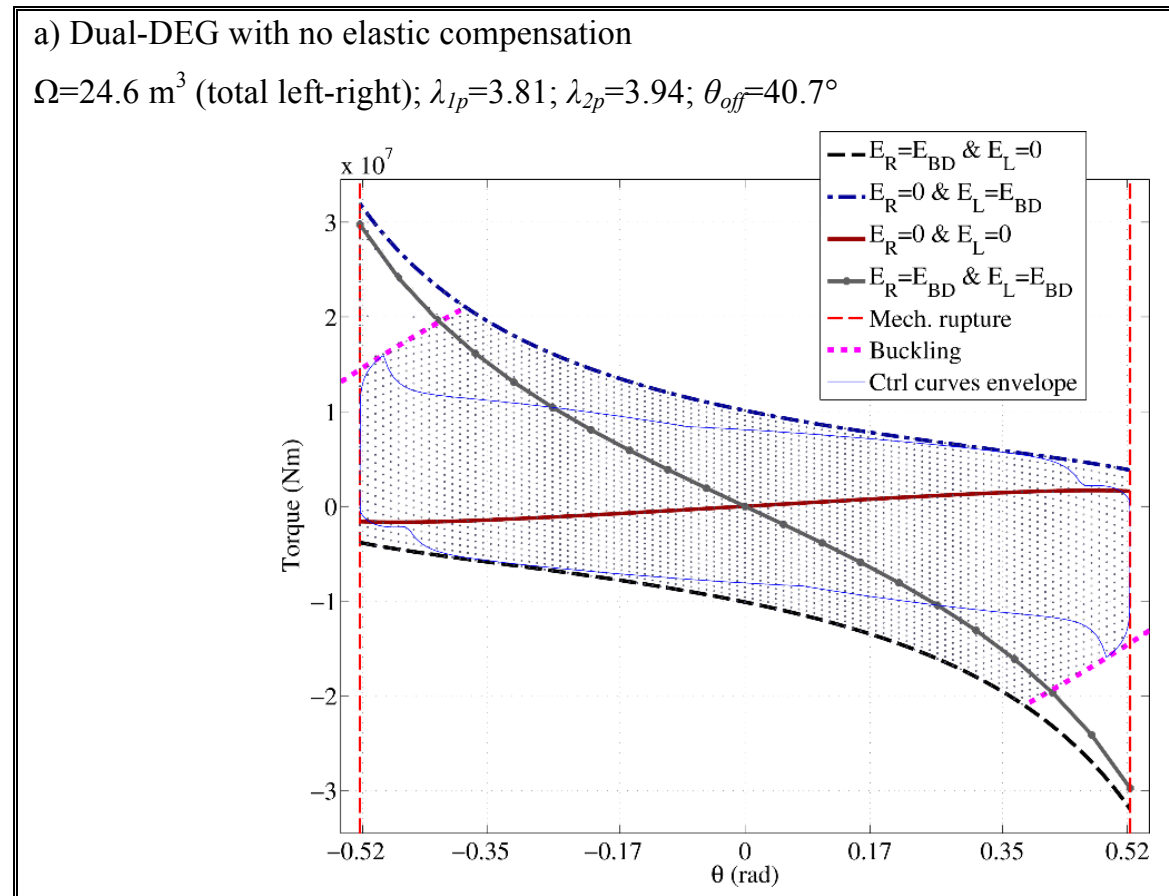

b) Dual-DEG with optimal elastic compensation

$\Omega=15.0 \mathrm{~m}^{3}$ (overall); $\lambda_{l p}=6.72 ; \lambda_{2 p}=3.91 ; \theta_{\text {off }}=49.3^{\circ} ; k_{S}=-1.40 \mathrm{e} 8 \mathrm{Nm}$

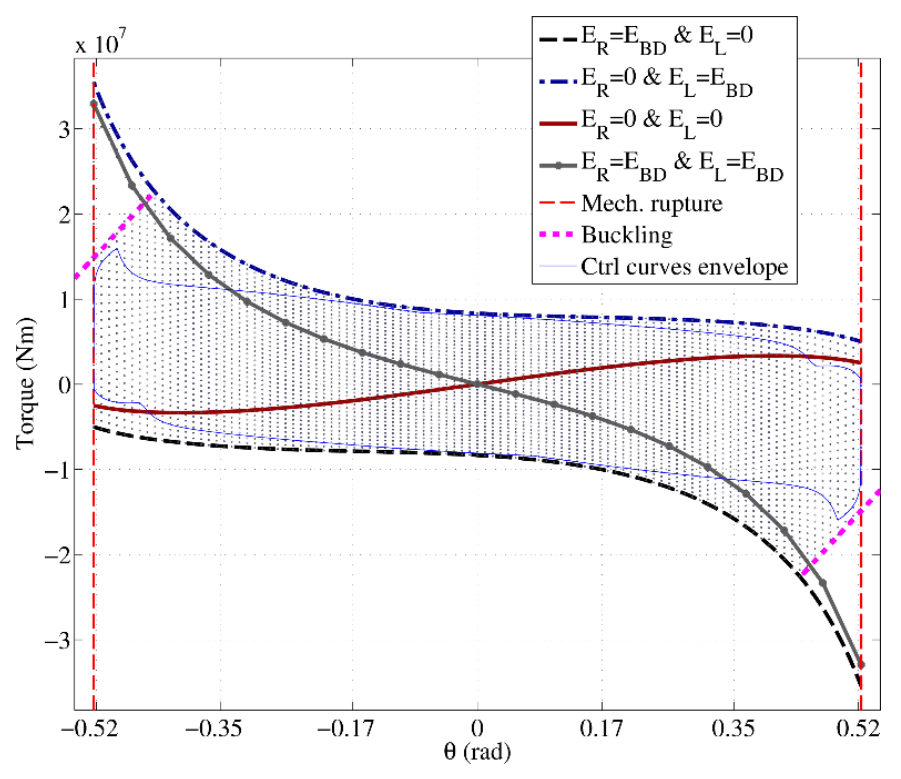

Figure 12 - PS-DEGs parameters optimization a) without external stiffness compensation and b) with external stiffness compensation. PS-DEG = Parallelogram Shaped Dielectric Elastomer Generator. 
In particular, Figure 12.b reports the results for the case where the additional passive spring is considered. In this case, the plotted Dual-DEG PTO characteristic also includes the contribution of the passive spring. As shown, the optimal value of $k_{S}$ is negative. A discussion on how such a negative stiffness spring could be implemented is deferred to a subsequent section.

Notice that, in order to envelope a given control trajectory, the electric field control is required to activate the DEGs even when their capacitance is increasing (i.e, the DEGs are required to operate as actuators also, not only as generators, during a significant part of their operation).

As regards the adequate exploitation of the employed DE material in the considered application, a convenient index to be used is the "cycle energy density", which is defined as the energy harvested in a cycle by a unit volume (or mass) of active DE material. For the cases with and without the passive compensation spring, the cycle energy density results in $9.66 \cdot 10^{5} \mathrm{~J} / \mathrm{m}^{3}\left(0.966 \mathrm{~kJ} / \mathrm{kg}\right.$, assuming $1000 \mathrm{~kg} / \mathrm{m}^{3}$ of rubber density) and $5.89 \cdot 10^{5} \mathrm{~J} / \mathrm{m}^{3}(0.589 \mathrm{~kJ} / \mathrm{kg})$.

\subsection{PS-DEG Control Cycles}

In this section, we describe how the electric field on the two agonist-antagonist PS-DEGs should be controlled in order to realise the desired control trajectory.

To obtain a given force-displacement response, a value for $E_{R}$ and $E_{L}$ must be identified for each configuration of the flap. In a given configuration, the same value of $T_{P T O}$ can be realized with different choices of the couple $\left(E_{R}, E_{L}\right)$. Here, a reciprocal activation strategy is used so that only the left DEG is activated (thus, $\left.E_{R}=0\right)$ in case the required PTO torque is higher than the purely elastic torque provided by the Dual-DEG in the considered configuration, and vice versa. This choice is made since it allows to use lower electric field values. Once the $T_{\text {PTO }}$ profile is known, the electric field on the active PS-DEG can be calculated, for any given value of $\theta$, by using equations (20) and (25).

Further than the description in the $\theta-T_{\text {PTO }}$ plane, the control cycle of the PS-DEGs can also be conveniently represented on the charge-voltage $(Q-V)$ plane [7], which provides useful information on how to properly command the energy harvesting cycles.

As already mentioned, PS-DEG charge and voltage are related to $\theta$ and $E$ via equations (13). Therefore, knowing $\theta$ and $E$ during a full oscillation, it is possible to trace the corresponding $Q-V$ cycle.

Energy harvesting cycles on the $Q-V$ plane identify the electric variables at any intermediate configuration during a single oscillation of a DEG. Each point of the cycle is associated to a specific configuration of the DEG, identified by the value of the capacitance, which is the ratio of the charge and the voltage in the considered point of the cycle.

To provide a general formulation, introduce the dimensionless charge, $Q^{*}$, and voltage, $V^{*}$, as follows:

$$
Q^{*}=\frac{Q}{\varepsilon E_{B D} l_{1} l_{2}}, V^{*}=\frac{V}{E_{B D} \Omega /\left(l_{1} l_{2}\right)} .
$$



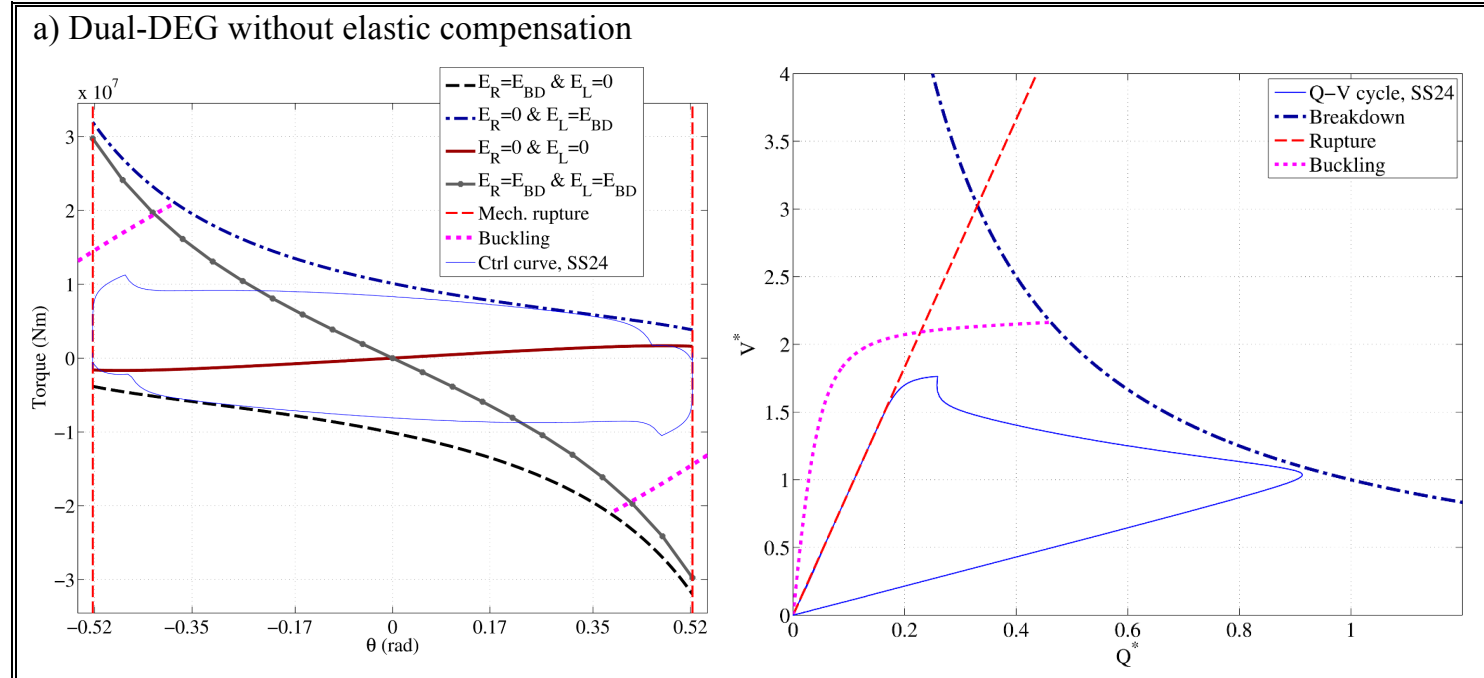

b) Dual-DEG with elastic compensation
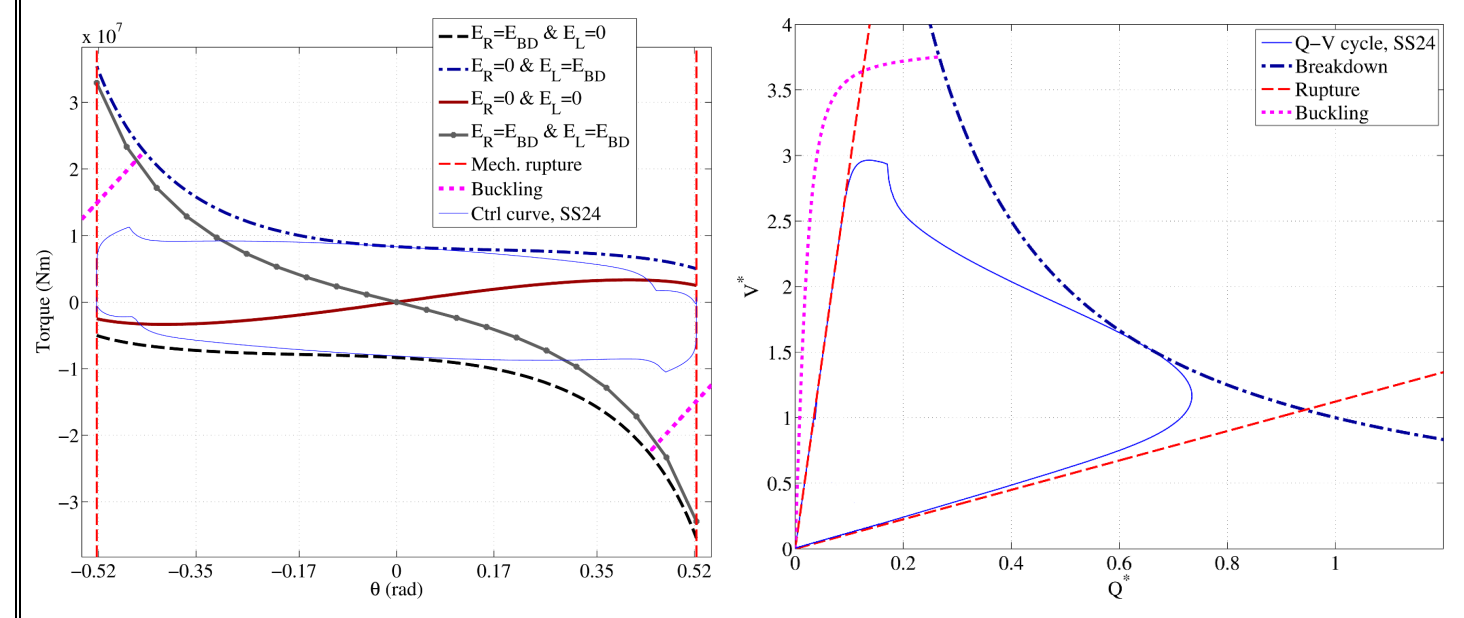

Figure 13 - Control trajectories for the SS24 on the $\theta-T_{\text {РТО }}$ plane and on the dimensionless $Q-V$ plane, for the case a) without and b) with external torsion spring compensation.

In Figure 13, the $\theta-T_{P T O}$ characteristic (on the left) and the corresponding dimensionless $Q-V$ cycle (on the right) are reported for both the design alternatives (that is, without elastic compensation, Figure 13.a, and with elastic compensation, Figure 13.b) in case of sea state SS24. In the same pictures, the curves representing the DE membrane failure mechanisms are reported in differently coloured dotted lines. Notice that, the reported $Q-V$ cycle is the same for the two PS-DEGs of the agonistic-antagonistic generator.

\section{Discussion}

In this section, the numerical results of the reported case study are compared with those of previous works, and general considerations on Dual-DEG PTO design and operation are outlined.

In an earlier paper [13], a non-optimal control approach was investigated for an identical flap provided with a single PS-DEG PTO as depicted in Figure 1.a and operating in the same reference location. With such PTO and controller, the energy productivity over 1 year of continuous operation resulted in $1.8 \mathrm{GWh}$ and required $44 \mathrm{~m}^{3}$ of DE material for DEG PTO implementation. With respect to that preliminary work, the results presented in this article provide a significant improvement, demonstrating that with the dual-DEG configuration a larger amount of electrical energy can be generated $(2.79 \mathrm{GWh})$ with a reduced amount of DE material (as low as $15 \mathrm{~m}^{3}$ ). 
In this analysis, the polymeric PTO has been dimensioned including all of the reference sea states in the design procedure. However, as can be understood from Figure 11, preventing Poly-Surge operation (for instance, by keeping it in a security position) in presence of certain sea wave conditions (namely, those that are very powerful but have low occurrence frequency) makes it possible to significantly change the required PTO characteristic. In general, this is likely to bring to a reduction of the amount of DE material required for PTO implementation. A more comprehensive analysis should take into account these aspects by properly comparing the advantage of requiring less DE material usage with the corresponding disadvantage of extracting a lower amount of energy. The results of numerical simulations showed that the introduction of a negative stiffness in parallel to the DualDEG PTO may allow to save a significant amount of DE material. For the examined system architecture, a negative torsion stiffness can be practically implemented by inserting a positive linear spring between joints $A$ and $C$ of the parallel mechanism depicted in Figure 4.b. In fact, assuming a spring with zero rest length and elastic constant $k_{L}$, the resulting torque contribution on the flap, $T_{S}$, is given by:

$T_{S}=-2 k_{L} l_{1} l_{2} \sin \theta_{\text {off }} \sin \theta$

Considering that $\theta$ varies in a relatively small interval around the position $\theta=0$, such an elastic torque can be approximated by $T_{S} \approx-k_{S} \theta$, with $k_{S}$ being

$k_{S}=2 k_{L} l_{1} l_{2} \sin \theta_{o f f}$.

Equation (28) can be used to select a proper value of $k_{L}$.

It must be remarked that, although the employment of external springs allows a reduction in DE material usage, their addition requires a further expense for spring purchase and installation. As above, in practical situations, cost considerations will have to be included to select the optimal system architecture.

The choice of the reference elastomer is also important as it determines the needed amount of DE material. NR has been demonstrated to be interesting for this application, featuring large break-down electric field and presenting large stiffness, which helps in preventing DE membrane buckling.

Beyond the numerical results of this paper, other relevant issues that will be considered in future works are:

- the performance of the Poly-Surge concept in real-sea conditions, featured by poly-chromatic waves described by appropriate spectral models;

- the inclusion in the PS-DEG model of dissipative effects accounting for DE material viscoelasticity and electrical conductivity;

- the fatigue lifetime of the employed DE materials under the particular loading conditions that occur in DEGs (large strains, cyclical application of large electric fields);

- the degradation of DEG performance due to aging under marine environmental conditions.

These aspects require dedicated experimental investigations, and their importance must be verified with a techno-economic analysis capable of assessing Poly-Surge feasibility in relation to both its energy performance and its capital and operating costs.

\section{Conclusions}

This paper presents a concept of surging Wave Energy Converter (WEC), named Poly-Surge, that is designed to operate in shallow water (about $10 \mathrm{~m}$ depth). The Poly-Surge has a bottom hinged pitching flap as the primary mechanical interface with the waves and is equipped with a Dual Dielectric Elastomer Generator (Dual-DEG) Power Take-Off (PTO) system for mechanical-to-electrical energy conversion. Specifically, the considered PTO 
consists of two Parallelogram-Shaped DEGs (PS-DEGs) arranged in an agonist-antagonist configuration, which enables to provide the flap with a bidirectional controllable torque.

Two mathematical models for Poly-Surge systems have been developed: one for the hydrodynamics of the primary interface (namely, the surging flap), which relies on potential flow theory and accounts for motion constrains, and one for the electro-elastic response of the Dual-DEG, which assumes a non-dissipative hyperelastic and linear dielectric behaviour of the employed elastomeric materials.

The considered models are then used to investigate the potentialities of the Poly-Surge concept and to design its Dual-DEG PTO. In particular, the developed hydrodynamics model is first used to determine the optimal control curves that should be provided by a PTO to maximize energy extraction in a site characterized by a specific climate. Second, the electro-elastic model is used to find the optimal parameters of a Dual-DEG that makes it possible to realize the desired PTO control curves.

To provide an example, a numerical case study is presented for a flap of given realistic dimensions and for an existing location with known wave climate. Results demonstrated that a practical Poly-Surge system can generate an electric power outputs of up to $1.56 \mathrm{MW}$ with a required amount of elastomeric material as low as 15 $\mathrm{m}^{3}$. As compared to a previous preliminary study [13], which considered a Poly-Surge system equipped with a single PS-DEG, energy productivity is increased by $55 \%$ and the amount of required elastomeric material is reduced by $65 \%$. The following must be remarked: the numerical results presented in this paper are affected by significant uncertainty due to the simplifications introduced in the models. Nevertheless, the order of magnitude of electric power output and required elastomeric material confirm that Poly-Surge is an interesting and potentially advantageous WEC concept.

Future works may include a techno-economic analysis of the Poly-Surge concept, which would also account for technological issues like the durability and survivability of the polymeric PTO, as well as experiments on small scale prototypes, which require to be tested in specific infrastructures (wave flumes or wave tanks).

\section{Acknowledgements}

The work presented in this paper is developed in the context of the project PolyWEC (www.polywec.org), a FP7FET- Energy project. The research leading to these results has received funding from the European Union Seventh Framework Programme [FP7/2007-2013] under grant agreement nº 309139.

The authors sincerely thank Dr. David Forehand (Edinburgh University) for providing the hydrodynamic outputs from WAMIT, and Eng. Antonello Cherubini (Scuola Sant'Anna, Pisa) for patiently reviewing the manuscript.

\section{References}

[1] Salter, S. H. (1974). Wave power. Nature, 249(5459), 720-724.

[2] Falcão, A. (2010). Wave energy utilization: A review of the technologies. Renewable and sustainable energy reviews, 14(3), 899-918.

[3] Falnes, J. (2007). A review of wave-energy extraction. Marine Structures, 20(4), 185-201.

[4] Drew, B., Plummer, A. R., Sahinkaya, M. N. (2009). A review of wave energy converter technology. Proceedings of the Institution of Mechanical Engineers, Part A: Journal of Power and Energy, 223(8), $887-902$. 
[5] Carpi, F., De Rossi, D., Kornbluh, R., Pelrine, R. E., Sommer-Larsen, P. (2011). Dielectric elastomers as electromechanical transducers: Fundamentals, materials, devices, models and applications of an emerging electroactive polymer technology. Elsevier.

[6] Pelrine, R., Kornbluh, R. D., Eckerle, J., Jeuck, P., Oh, S., Pei, Q., Stanford, S. (2001). Dielectric elastomers: generator mode fundamentals and applications. In SPIE's 8th Annual International Symposium on Smart Structures and Materials (pp. 148-156).

[7] Koh, S.J., Keplinger, C., Li, T., Bauer, S., Suo, Z. (2011). Dielectric elastomer generators: How much energy can be converted?. IEEE/ASME Transactions on Mechatronics, 16(1), 33-41.

[8] Chiba, S., Waki, M., Kornbluh, R., Pelrine, R. (2008). Innovative power generators for energy harvesting using electroactive polymer artificial muscles. In The 15th International Symposium on: Smart Structures and Materials Nondestructive Evaluation and Health Monitoring (pp. 692715692715). International Society for Optics and Photonics.

[9] Jean, P., Wattez, A., Ardoise, G., Melis, C., Van Kessel, R., Fourmon, A., Barrabino, E., Heemskerk, J., Queau, J. P. (2012). Standing wave tube electro active polymer wave energy converter. In SPIE Smart Structures and Materials+ Nondestructive Evaluation and Health Monitoring (pp. 83400C-83400C). International Society for Optics and Photonics.

[10] Babarit, A., Gendron, B., Singh, J., Mélis, C., Jean, P. (2013). Hydro-Elastic Modelling of an ElectroActive Wave Energy Converter. In ASME 2013 32nd International Conference on Ocean, Offshore and Arctic Engineering (pp. V009T12A033-V009T12A033). American Society of Mechanical Engineers.

[11] Rosati Papini, G. P., Vertechy, R., Fontana, M. (2013). Dynamic Model of Dielectric Elastomer Diaphragm Generators for Oscillating Water Column Wave Energy Converters. In ASME 2013 Conference on Smart Materials, Adaptive Structures and Intelligent Systems (pp. V001T03A038V001T03A038). American Society of Mechanical Engineers.

[12] Scherber, B., Grauer, M., Köllnberger, A. (2013). Electroactive polymers for gaining sea power. In SPIE Smart Structures and Materials+ Nondestructive Evaluation and Health Monitoring (pp. 86870K86870K). International Society for Optics and Photonics.

[13] Moretti, G., Forehand, D., Vertechy, R., Fontana, M., Ingram, D. (2014). Modeling Of an Oscillating Wave Surge Converter With Dielectric Elastomer Power Take-Off. In Proc. of the ASME 2014 33rd International Conference on Ocean, Offshore and Arctic Engineering, OMAE2014-23559.

[14] Whittaker, T., Folley, M. (2012). Nearshore oscillating wave surge converters and the development of Oyster. Philosophical Transactions of the Royal Society A: Mathematical, Physical and Engineering Sciences, 370(1959), 345-364.

[15] Moretti, G., Fontana, M., Vertechy, R. (2014). Parallelogram-shaped dielectric elastomer generators: analytical model and experimental validation. Journal of Intelligent Material Systems and Structures, in press (Manuscript ID JIM-14-054).

[16]Folley, M., Whittaker, T. J. T. (2009). Analysis of the nearshore wave energy resource. Renewable Energy, 34(7), 1709-1715.

[17] Folley, M., Whittaker, T., Osterried, M. (2004). The oscillating wave surge converter. In Fourteenth International Offshore and Polar Engineering Conference. 
[18]Folley, M., Whittaker, T., Van't Hoff, J. (2007). The design of small seabed-mounted bottom-hinged wave energy converters. In Proceedings of the 7th European Wave and Tidal Energy Conference, Porto, Portugal.

[19] Babarit, A., Hals, J., Muliawan, M. J., Kurniawan, A., Moan, T., Krokstad, J. (2012). Numerical benchmarking study of a selection of wave energy converters. Renewable Energy, 41, 44-63.

[20] Pérez, T., Fossen, T. I. (2008). Time-vs. frequency-domain identification of parametric radiation force models for marine structures at zero speed. Modeling, Identification and Control, 29(1), 1-19.

[21] Falnes, J. (2002). Ocean waves and oscillating systems. Cambridge University Press.

[22] Hals, J., Falnes, J., Moan, T. (2011). Constrained optimal control of a heaving buoy wave-energy converter. Journal of Offshore Mechanics and Arctic Engineering, 133(1), 011401.

[23] Eidsmoen, H. (1996). Optimum control of a floating wave-energy converter with restricted amplitude. Journal of Offshore Mechanics and Arctic Engineering, 118(2), 96-102.

[24] Nolan, G. A., Ringwood, J. V., Leithead, W., Butler, S. (2005). Optimal damping profiles for a heaving buoy wave-energy converter. In Proceedings of the Fifteenth International Offshore and Polar Engineering Conference (ISOPE) (Vol. 1, pp. 477-485).

[25] Moretti, G., Fontana, M., Vertechy, R. (2014). Modeling of a Heaving Buoy Wave Energy Converter with Stacked Dielectric Elastomer Generator. In ASME 2014 Conference on Smart Materials, Adaptive Structures and Intelligent Systems. American Society of Mechanical Engineers.

[26] Holzapfel, G. A. (2000). Nonlinear solid mechanics (Vol. 24). Wiley \& Sons.

[27] Steinmann, P., Hossain, M., Possart, G. (2012). Hyperelastic models for rubber-like materials: consistent tangent operators and suitability for Treloar's data. Archive of Applied Mechanics, 82(9), 1183-1217.

[28] Hamdi, A., NaïtAbdelaziz, M., Ait Hocine, N., Heuillet, P., Benseddiq, N. (2006). A fracture criterion of rubber-like materials under plane stress conditions. Polymer Testing, 25(8), 994-1005.

[29] Ingram, D., Smith, G., Bittencourt-Ferreira, C., Smith, H. (2011). Protocols for the equitable assessment of marine energy converters. The University of Edinburgh, School of Engineering. 